\title{
Peripheral deep anterior lamellar keratoplasty using a cryopreserved donor cornea for Terrien's marginal degeneration*
}

\author{
Dan HUANG, Wen-ya QIU, Bei ZHANG, Bing-hong WANG, Yu-feng YAO \\ (Department of Ophthalmology, Sir Run Run Shaw Hospital, School of Medicine, Zhejiang University, Hangzhou 310016, China) \\ †E-mail: yaoyuf@mail.hz.zj.cn \\ Received Mar. 20, 2014; Revision accepted Aug. 30, 2014; Crosschecked Nov. 14, 2014
}

\begin{abstract}
Objective: To evaluate the clinical efficacy of peripheral deep anterior lamellar keratoplasty (DALK) using a cryopreserved donor cornea for Terrien's marginal degeneration (TMD). Methods: Thirty-one eyes of 27 patients with TMD underwent peripheral DALK using cryopreserved donor corneas. According to the distance between the inner edge of the lesion and the limbus, a ring-shaped or D-shaped DALK was performed. All grafts were stored at $-20^{\circ} \mathrm{C}$. Cryopreserved corneoscleral rims were prepared for ring-shaped grafts and cryopreserved whole eyeballs were prepared for D-shaped grafts. The general conditions, intraoperative performance, postoperative corneal reconstruction, astigmatism, best corrected visual acuity (BCVA), and various complications were analyzed. Results: Ring-shaped DALK was performed in 28 eyes and D-shaped DALK was performed in 3 eyes. Postoperative follow-up time was $(28.4 \pm 24.8)$ months. There was evidence of inflammation before surgery in 12 eyes $(38.7 \%)$ and intraoperative perforation occurred in 13 eyes $(41.9 \%)$. The corneal structures of all eyes were reconstructed. Postoperative astigmatism and BCVA showed improvement (both $P=0.00$ ) except for cases that underwent $D$-shaped DALK. Ten eyes $(32.2 \%)$ developed transient ocular hypertension and one eye (3.2\%) developed secondary glaucoma. No primary disease recurrence or corneal allograft rejection was observed. Conclusions: Peripheral DALK for TMD using cryopreserved donor tissue is an effective technique that eliminates rejection and extends the use of donor eyes. Inflammatory history or intraoperative perforation has no adverse effect on graft recovery. However, D-shaped DALK did not achieve good visual outcomes.
\end{abstract}

Key words: Terrien's marginal degeneration (TMD), Deep anterior lamellar keratoplasty (DALK), Cryopreservation doi: 10.1631 jzus.B1400083

Document code: A CLC number: R772

\section{Introduction}

Terrien's marginal degeneration (TMD) is an uncommon corneal disease characterized by progressive marginal thinning of the cornea, the aetiology and pathogenesis of which are unknown (Duke-Elder, 1965; Grayson, 1979). Two main forms of TMD are recognized: the classic form, which occurs mostly in patients older than 40 years and shows extremely

\footnotetext{
${ }^{\ddagger}$ Corresponding author

* Project supported by the Major Program for Science and Technology Research of Zhejiang Province (No. 2011C13029-2) and the Medical Scientific Research Foundation of Zhejiang Province (Nos. 2012ZDA026 and 2013ZDA012), China

(D) ORCID: Yu-feng YAO, http://orcid.org/0000-0003-1494-9711

(C) Zhejiang University and Springer-Verlag Berlin Heidelberg 2014
}

slow progression with no inflammation (Iwamoto et al., 1972; Guyer et al., 1987), and a variant form with prominent inflammatory signs, such as ocular surface hyperaemia, which occurs in the younger population (Austin and Brown, 1981). Typically, these lesions begin superonasally and undergo progressive stromal thinning with opacification, superficial vascularization, and lipid deposition at the central edge. This thinning progresses circumferentially and, in rare cases, centrally. In advanced cases, extreme thinning causes local cornea ectasia and protrusion, which may result in marked irregular astigmatism and poor visual acuity. Perforation occurs spontaneously or after minor trauma in approximately $15 \%$ of TMD cases (Duke-Elder, 1965; Grayson, 1979). Therefore, surgical management should be considered in cases of 
severe astigmatism, perforation, or impending perforation to reconstruct corneal structure, regain normal corneal curvature, and improve visual outcomes.

To date, few studies pertaining to the surgical treatment of TMD have been reported. Although different surgical techniques have been tested to treat peripheral corneal diseases over the years, peripheral lamellar keratoplasty (LK) is regarded as the most acceptable treatment (Brown et al., 1983; Guyer et al., 1987; Pettit, 1991; Cheng et al., 2005; Huang et al., 2008; Wang et al., 2012). Conventionally, the peripheral LK graft is obtained from a whole fresh eyeball, which has an allograft rejection rate of 10\%-14\% (Caldwell et al., 1984; Pettit, 1991). Some anti-rejection medications, such as cyclosporine A, have been administered following the LK operations, but these are known to have potentially serious systemic side effects (Wang et al., 2012).

We have previously reported the advantages of cryopreserved donor corneal buttons for full-bed deep lamellar keratoplasty (FBDLK) grafting with favorable corneal clarity and no rejection (Yao et al., 2002; 2006; Yao, 2008). After central corneal transplantation, different diameters of residual corneoscleral rims remain, and these residual corneoscleral rims can be used in peripheral keratoplasty.

Herein, we report a series of peripheral deep anterior lamellar keratoplasty (DALK) for TMD using cryopreserved donor tissue. To the best of our knowledge, this is the first case series analyzing the applicability of using cryopreserved donor tissue in peripheral DALK for TMD.

\section{Patients and methods}

\subsection{Patients}

From June 1998 to December 2012, 31 eyes from 27 patients with TMD underwent peripheral DALK surgery in the cornea clinic at the Sir Run Run Shaw Hospital, School of Medicine, Zhejiang University, Hangzhou, China. The diagnosis of TMD was made on the basis of typical peripheral corneal thinning, opacification, vascularization, and lipid deposition. Indications for surgery included peripheral corneal perforation, Descemet's membrane bulging, or thickness at the thinnest point no more than one quarter of the normal corneal thickness with corneal ectasia. Signs of ocular surface hyperaemia were eliminated with eyedrops before surgery for patients with inflammatory type TMD. Patients receiving postoperative follow-up of fewer than six months were excluded.

\subsection{Surgical methods}

All surgery was performed by the same surgeon (YFY). If the lesion progressed circumferentially and the inner edge was in the peripheral section without involving the pupil area, ring-shaped DALK was selected; if the lesion progressed both circumferentially and centrally and the inner edge was close to or involving the pupil area, D-shaped DALK was selected.

All surgery was performed while the patient was under general anesthesia. After the upper and lower rectus muscles were fixed, the conjunctiva adjacent to the diseased cornea was cut in a way to save limbal stem cells at the conjunctival edge. Next, the conjunctiva was retracted from the limbus and the subconjunctival tissue was excised to expose the bare sclera. For ring-shaped DALK, a trephine was used to mark the inner edge of the corneal lesion, with an inner diameter $0.5 \mathrm{~mm}$ smaller than the lipid edge to ensure that the entire degenerative cornea was involved. A diamond knife was then employed to deepen the trephine groove. The outer edge was $0.5 \mathrm{~mm}$ larger than the thinned corneal limbus and was marked by a freehand incision using a diamond knife parallel to the clear arc of the limbus. Two ends of the ring were outlined by a freehand incision (Figs. 1a and 1b). For D-shaped DALK, the inner edge was defined as the line incised freehand between the two points on either side of the lesion, and the outer edge was also marked parallel to the arc of the limbus (Figs. 1c and 1d). Following the outline, the stroma, including lipid deposition, was removed almost to the level of Descemet's membrane. If there was a large perforation, a postoperative shallow anterior chamber may occur. Iridectomy was performed to prevent postoperative papillary block and secondary glaucoma. After iridectomy was finished, the lamellar dissection was continued.

All corneal donor tissue was stored at $-20{ }^{\circ} \mathrm{C}$. Routinely, we perform FBDLK or penetrating keratoplasty (PK) with a trephine of 7.25 to $8.25 \mathrm{~mm}$ in diameter, and for these cases the retained peripheral corneoscleral rims were also stored at $-20{ }^{\circ} \mathrm{C}$. If we 


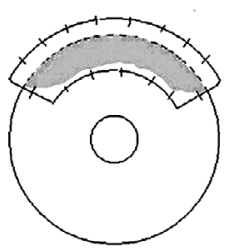

(a)

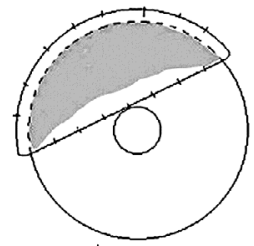

(c)

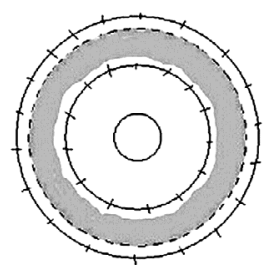

(b)

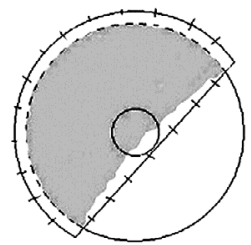

(d)
Fig. 1 Dimensions of the corneoscleral bed and graft (a) If the lesion was in the peripheral part and partially involved the perimeter, a partial ring-shaped DALK was chosen. (b) If the lesion was in the peripheral part and involved the entire perimeter, a complete ring-shaped DALK was chosen. (c) If the inner edge of the lesion was close to the pupil area, a small D-shaped DALK was chosen and sutures did not cross the pupil. (d) If the inner edge of the lesion involved the pupil area, a large D-shaped DALK was chosen and the sutures did not cross the pupil

performed ring-shaped DALK, the donor graft was obtained from the peripheral corneoscleral rim. Before surgery, the lesion was measured and the matched cryopreserved donor corneoscleral rim with the same inner diameter was selected. During surgery, the outer edge and the two ends of the graft were further trimmed to match the bed. If we performed D-shaped DALK, the cryopreserved whole eyeball was prepared. During surgery, the edge was incised using a diamond knife, implementing the same method as when the bed was marked. Next, the full-thickness graft was reduced in size using scissors. After the epithelium and endothelium were wiped away, the full-thickness graft was sutured in place with interrupted and tight sutures, using 10-0 nylon for compressive sutures.

\subsection{Postoperative medication and follow-up}

Routinely, the patients were administered $0.3 \%$ ofloxacin or $0.5 \%$ levofloxacin (Santen Pharmaceutical Co., Osaka, Japan) and $0.1 \%$ fluorometholone (Santen Pharmaceutical Co.) eyedrops four times daily for six months. In addition, intravenous $20 \%$ mannitol $250 \mathrm{mg}$ once was added immediately after the operation if the peripheral graft was relatively large. If intraocular pressure (IOP) increased, topical anti-glaucoma eyedrops were added. All patients were followed up on a weekly basis for one month and monthly for six months, and then at different intervals. Indications for selective suture removal included excessive tightness or looseness, exposure, vascularization, or astigmatism.

\subsection{Major measurements}

Major measurements included general characteristics of the patients, intraoperative performance, postoperative reconstruction of the corneal structure, corneal astigmatism, visual acuity, and surgical complications. Lipid deposition was graded as none, slight, moderate, or marked based on its circumferential extension and involving depth by visual inspection. Corneal reconstruction was accessed by slitlamp photographs and anterior segment optical coherence tomography (AS-OCT; Visante, Carl Zeiss Meditec, Dublin, California, USA). Graft clarity was graded as transparent, slightly opaque (partially obscuring iris details), or obviously opaque (completely obscuring all details of intraocular structures). Snellen best corrected visual acuity (BCVA) was recorded and converted to the base-10 logarithm of the minimum angle of resolution (lgMAR) for calculating mean and standard deviation.

High IOP complications were defined as eyes with normal preoperative IOP but with IOP over $21 \mathrm{mmHg}$ postoperatively. Secondary glaucoma was defined as eyes with uncontrollably high IOP needing continuous topical anti-glaucoma medication for more than three months or trabeculectomy for controlling eye pressure. Graft rejection was defined as the development of an epithelial or endothelial rejection line or characteristic keratic precipitates, or spreading corneal oedema with cell infiltration in the stroma in a previously clear graft. Complicated cataract was diagnosed when the crystal lens turned opaque postoperatively. These definitions have also been described by our previous report (Wu et al., 2012).

\subsection{Statistical analysis}

All datasets were tested for normality using the Kolmogorov-Smirnov test. The Pearson Chi-square test, independent $t$-test, and Mann-Whitney $U$ tests 
were used to investigate differences in categorical variables, normally distributed and non-normally distributed continuous variables, respectively. Linear regression was used to determine the relationship between high IOP and factors. All tests were two-tailed and a $P$ value of less than 0.05 was deemed statistically significant.

\section{Results}

\subsection{General characteristics}

Thirty-one eyes from 27 patients were included in this series, 16 males and 11 females, with a mean age of $(45.9 \pm 13.9)$ years. The binocular morbidity rate was $70 \%(19 / 27)$. There were 12 patients who had a history of recurrent ocular surface inflammation. Median follow-up time was $(28.4 \pm 24.8)$ months (range, 6-110 months).

Based on a history of inflammation, the eyes were divided into the inflammatory group (12 eyes) and the noninflammatory group (19 eyes). The mean age was $(43.9 \pm 10.2)$ years in the inflammatory group and $(47.1 \pm 14.3)$ years in the noninflammatory group $(P=0.18)$. The mean clock hours of the disease area was $(8.3 \pm 3.5) \mathrm{h}$ in the inflammatory group and $(5.2 \pm 1.5) \mathrm{h}$ in the noninflammatory group $(P=0.004)$. Lipid deposition in the inflammatory group (Fig. 2a) was greater $(P=0.01)$ than that in the noninflammatory group (Fig. 2b).
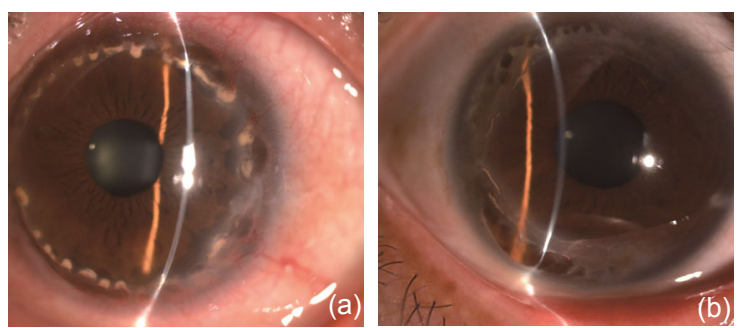

Fig. 2 Characteristics of the inflammatory (a) and noninflammatory (b) TMDs

(a) Inflammatory TMD: the patient complained of recurrent redness, pain, and photophobia for more than 5 years. Slitlamp microscopy showed ocular surface hyperaemia, entire peripheral cornea thinning, and lipid deposition involving the entire ring and deep stroma. (b) Noninflammatory TMD: the patient complained of decreased visual acuity for 20 years but no redness or pain. Slit-lamp microscopy showed no hyperaemia and lipid deposition involved only a small part of the peripheral cornea and superficial stroma

\subsection{Intraoperative performance}

During the operations, perforation occurred in 13 eyes $(41.9 \%)$, showing that the clear aqueous humor effused immediately after the epithelium was removed. There were four eyes with a history of perforation before surgery, which subsequently healed. Of the nine eyes with no history of perforation, perforation in four eyes was evident in the thinnest point of the ectasia cornea and appeared as a hole, while perforation in the other five eyes was in the leading edge of lipid deposition with a crack-like appearance. The incidence of perforation was $66.6 \%$ $(8 / 12)$ in the inflammatory group and $26.3 \%(5 / 19)$ in the noninflammatory group $(P=0.02)$.

\subsection{Reconstruction of the corneal structure}

Reconstruction of the corneal structure was successfully completed in all eyes. All grafts achieved complete corneal epithelialization in 3-7 d, depending on the size of the graft. A double anterior chamber did not occur in any of the perforated eyes. Because cryopreserved donor corneas were used, the graft was comparatively oedematous in the early postoperative period and then gradually clarified. Generally, the oedema was completely gone after 2-3 months (Fig. 3). Three months after surgery, slit-lamp microscopy and AS-OCT showed that all grafts were well attached to the recipient beds, the peripheral corneas restored to normal thickness, and all cases preserved anatomic integrity (Fig. 4). The thickness of the graft was stable throughout the entire follow-up time and no recurrence of corneal thinning was observed (Fig. 4b). During the follow-up period, 5 grafts were transparent, 18 grafts were slightly opaque, and 8 grafts were obviously opaque. In the perforated eyes, grafts were slightly opaque in seven eyes and obviously opaque in the other six eyes. Vascularization of the graft occurred in 12 eyes $(38.7 \%)$. In the inflammatory group, no inflammation recurrence was observed after the operation.

\subsection{Astigmatism and visual acuity}

According to the clock hours of the disease area, the eyes that underwent ring-shaped DALK were allocated into one of four groups: group $1, \leq 1$ quadrant ( 3 eyes); group $2,>1$ quadrant and $\leq 2$ quadrants (14 eyes); group $3,>2$ quadrants and $\leq 3$ quadrants 
( 6 eyes); and group 4,>3 quadrants ( 5 eyes). Group 5 included the 3 eyes that underwent D-shaped DALK.
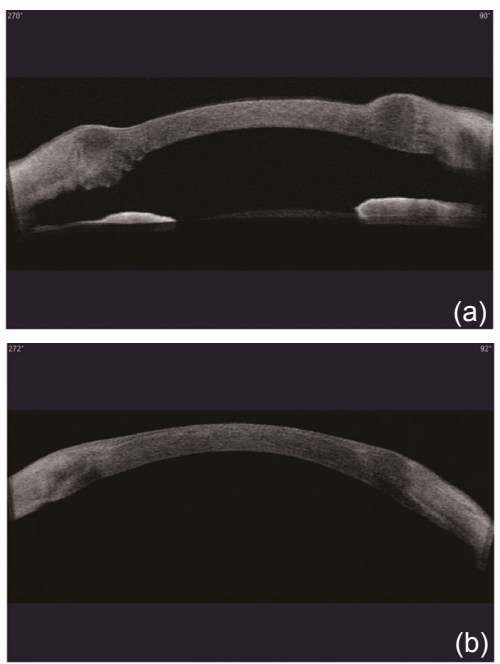

Fig. 3 AS-OCT images of the grafts

AS-OCT showed oedema of the peripheral grafts. (a) The graft was obviously oedematous in one week after the operation. (b) The oedema of the graft was completely resolved in two months after the operation

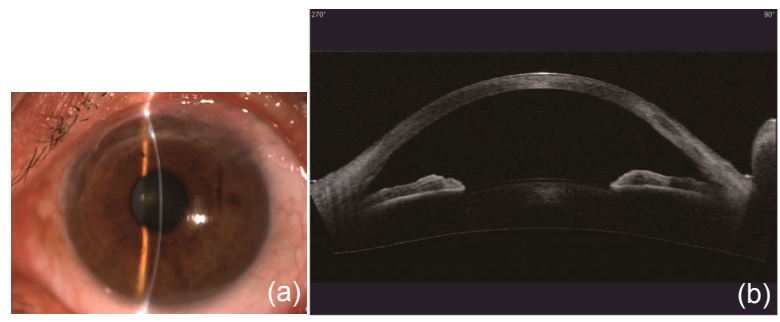

Fig. 4 Reconstruction of the corneal structure

Slit-lamp microscopy (a) and AS-OCT image (b) show that the graft attached well to the recipient bed and the thickness of the graft remained stable after 62 months postoperatively

As determined by a slit-lamp and AS-OCT, all corneas regained relatively normal curvature after six months. Fig. 5 showed that the corneal curvatures were steep preoperatively and back to normal postoperatively in two cases. The average preoperative astigmatism of all cases was $(6.80 \pm 3.91) \mathrm{D}$ and the postoperative astigmatism was $(3.58 \pm 2.21) \mathrm{D}$, which were statistically different $(P=0.01)$. In the three D-shaped DALK cases, the average preoperative astigmatism was $(8.00 \pm 4.58) \mathrm{D}$ and the postoperative astigmatism was $(7.31 \pm 3.04) \mathrm{D}$, which were not statistically different $(P=0.28$; Table 1$)$.
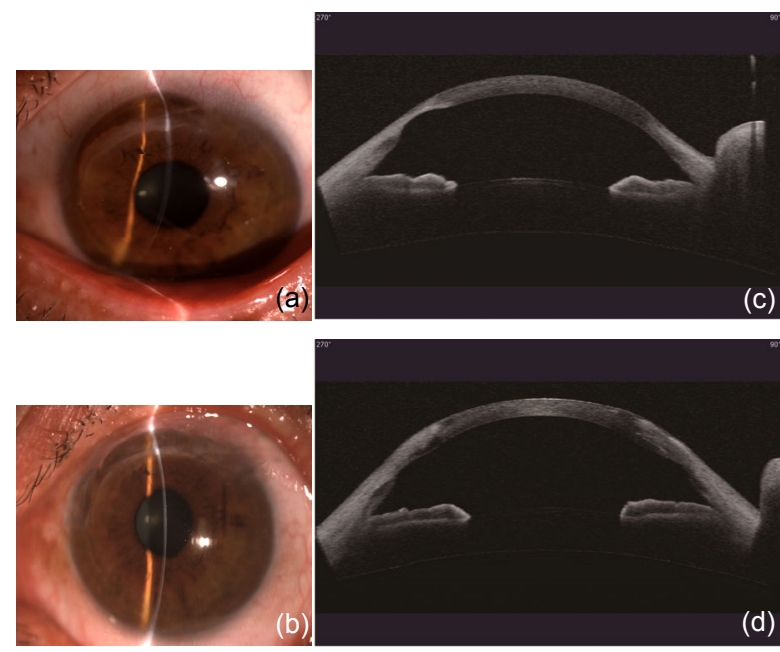

Fig. 5 Curvature of preoperative and postoperative corneas

(a, b) Slit-lamp microscopy showing corneal curvature preoperatively and 5 years postoperatively. (c, d) AS-OCT images from another patient showing corneal curvature preoperatively and 1 year postoperatively

Table 1 Comparison between preoperative and postoperative astigmatisms

\begin{tabular}{ccccc}
\hline \multirow{2}{*}{ Group } & \multirow{2}{*}{$\begin{array}{c}\text { Number } \\
\text { of eyes }\end{array}$} & \multicolumn{2}{c}{ Astigmatism (D) } & \\
\cline { 3 - 4 } & & Preoperative & Postoperative & \\
\hline 1 & 3 & $4.50 \pm 0.86$ & $2.41 \pm 2.09$ & 0.18 \\
2 & 14 & $7.41 \pm 5.05$ & $3.83 \pm 1.83$ & $0.01^{*}$ \\
3 & 6 & $5.94 \pm 1.20$ & $2.21 \pm 1.09$ & $0.02^{*}$ \\
4 & 5 & $5.92 \pm 2.92$ & $2.75 \pm 1.71$ & $0.04^{*}$ \\
5 & 3 & $8.00 \pm 4.58$ & $7.31 \pm 3.04$ & 0.28 \\
Total & 31 & $6.80 \pm 3.91$ & $3.58 \pm 2.21$ & $0.00^{*}$ \\
\hline${ }^{*} P<0.05$ & & & &
\end{tabular}

The mean postoperative BCVA of all eyes was statistically better than the preoperative counterparts $(P=0.00)$. In group 1 , in which the lesions occupied less than one quadrant, both preoperative and postoperative BCVAs were good, and there was no statistical difference $(P=0.42)$. In groups 2,3 , and 4 , postoperative BCVA was significantly better than preoperative BCVA $(P=0.00,0.01$, and 0.01 , respectively). In the D-shaped group, both preoperative and postoperative BCVAs were poor and similar $(P=1.0)$. Twenty-two eyes $(71 \%)$ achieved a postoperative BCVA of 0.5 or better (Table 2).

\subsection{Complications}

After the operations, high IOP occurred in 11 of $31(35.4 \%)$ eyes, of which 10 eyes returned to a 
Table 2 Comparison between preoperative and postoperative BCVAs

\begin{tabular}{|c|c|c|c|c|c|c|}
\hline \multirow{2}{*}{ Group } & \multirow{2}{*}{$\begin{array}{l}\text { Number } \\
\text { of eyes }\end{array}$} & \multicolumn{2}{|c|}{$\operatorname{lgMAR}$} & \multirow{2}{*}{$P$-value } & \multirow{2}{*}{$\begin{array}{c}\text { Row of } \\
\text { improvement }\end{array}$} & \multirow{2}{*}{$\begin{array}{c}\text { Postoperative BCVA } \\
\geq 0.5(\%)\end{array}$} \\
\hline & & Preoperative BCVA & Postoperative BCVA & & & \\
\hline 1 & 3 & $0.10 \pm 0.11$ & $0.07 \pm 0.12$ & 0.42 & 0.7 & $100.0(3 / 3)$ \\
\hline 2 & 14 & $0.70 \pm 0.53$ & $0.23 \pm 0.19$ & $0.00^{*}$ & 3.1 & $71.4(10 / 14)$ \\
\hline 3 & 6 & $0.88 \pm 0.59$ & $0.18 \pm 0.18$ & $0.01^{*}$ & 3.8 & $83.3(5 / 6)$ \\
\hline 4 & 5 & $0.72 \pm 0.24$ & $0.13 \pm 0.21$ & $0.01^{*}$ & 3.8 & $80.0(4 / 5)$ \\
\hline 5 & 3 & $0.86 \pm 0.11$ & $1.01 \pm 0.85$ & 1.00 & 0.0 & $0.0(0 / 3)$ \\
\hline Total & 31 & $0.69 \pm 0.49$ & $0.25 \pm 0.38$ & $0.00^{*}$ & 2.8 & $71.0(22 / 31)$ \\
\hline
\end{tabular}

${ }^{*} P<0.05$. BCVA: best corrected visual acuity; lgMAR: base-10 logarithm of the minimum angle of resolution

normal level $72 \mathrm{~h}$ after medical treatment. High IOP persisted for three months in one eye and resulted in secondary glaucoma. The IOP of this patient was eventually controlled with anti-glaucoma medication. The eye with secondary glaucoma was completely ring-shaped and had an intraoperative perforation. After surgery, the graft was oedematous and the peripheral anterior chamber was shallow. In the 11 eyes with high IOP, 4 grafts were completely ring-shaped and another 7 grafts occupied more than 2 quarters. In addition, in the 11 eyes with high IOP, 9 had intraoperative perforations. Our data showed that postoperative high IOP was associated with the range of the graft $(P=0.039)$ and intraoperative perforation $(P=0.033)$. There were no observations of corneal allograft rejection, complicated cataracts, graft infections, or other complications.

\section{Discussion}

For severe TMD, no standardized surgical management has been established since the lesion is peripheral and can be manifested in various shapes. Over the years, a number of types of surgical procedures, such as cauterization, simple excision, conjunctival flap, PK, and peripheral LK have been described to treat peripheral corneal diseases (Doggart, 1930; Anderson, 1977; Jonas et al., 2001; Javadi et al., 2004; Huang et al., 2008). Originally, Terrien treated a patient by repeated cauterization of the ecstatic area, with the objective of reducing the astigmatism by cicatricial contraction (Doggart, 1930). However, this surgical method was never subsequently used. The simple excision of the abnormally thinned corneal stroma and suturing of the near-normal thickness edges was suggested, but this operation distorted the corneal structure and could not result in normal corneal curvature when the lesion was large enough (Caldwell et al., 1984; Javadi et al., 2004; Maccheron and Daya, 2012). Conjunctival flap surgery was not widely accepted because it could not reconstruct the cornea and even worse it would aggravate inflammation as conjunctival vessels were brought into the cornea and induced vascularization and pseudopterygium (Anderson, 1977; Alino et al., 1998). PK and LK are effective measures in terms of rescuing the compromised eyeball and reconstructing the anterior segment (Panda et al., 1999; Soong et al., 2000; Jonas et al., 2001; Hanada et al., 2008). In the long term, PK has an estimated $20 \%-38 \%$ rejection rate, and the rate is even higher in larger diameter or eccentric grafts close to the limbus (Peleyer et al., 1992; Inq et al., 1998). Varley et al. (1990) reported that the allograft rejection rate is as high as $64 \%$ in patients with pellucid marginal corneal degeneration treated by PK. Therefore, LK, with the advantages of satisfactory anatomical reconstruction and no risk of endothelial rejection, is regarded as the more ideal procedure (Shimazaki, 2000). However, if the graft is large enough to cover the entire cornea, the normal corneal tissue along the visual axis must be removed, and the vision can be affected when the graft-host interface becomes opaque. Thus, for peripheral corneal diseases, peripheral LK was widely used by most surgeons (Brown et al., 1983; Guyer et al., 1987; Pettit, 1991; Cheng et al., 2005; Huang et al., 2008; Wang et al., 2012; Kursiah, 2013).

The peripheral LK was commonly used for peripheral corneal diseases to preserve the integrity of the globe. However, in this study, we performed peripheral DALK rather than LK for treating severe 
TMD, showing several advantages through changing the surgical strategy. Histopathologically, TMD is a peripheral corneal stromal disease. In severe cases, the peripheral stromal thinning of the cornea is replaced by vascularized connective tissue and lipid deposits, leaving only the unhealthy epithelium, extremely thinning and degenerated stroma and Descemet's membrane (Iwamoto et al., 1972). Therefore, in severe cases, in preparing the bed, it is easy to remove by peeling off rather than cutting the unhealthy stroma from the bed, leaving completely or nearly completely the exposed Descemet's membrane of the bed. Moreover, for matching such bed, fullthickness of the donor graft is better than a partial lamellar graft due to it being easier for preparing and also being more regular and smoother in shape. More importantly, using the full-thickness of the donor graft for repairing the peripheral lesion area provides stronger reinforcement of the cornea. We believe that further study may prove the advantages of our surgical strategy in treating such corneal diseases.

Generally, the peripheral LK graft is usually obtained from whole fresh eyeballs. In the present study, we performed peripheral DALK using cryopreserved donor corneas and reconstruction of the corneal structure was successfully completed in all eyes. The advantages of using cryopreserved donor corneas were remarkable.

First of all, no allograft rejection episodes in any eyes were observed. This is attributed to that cryopreserved donor tissue was used for grafting. Our previous study reported the mechanism of no allograft rejection (Yao et al., 2002; 2006; Yao, 2008; Wu et al., 2012). Since we had scraped off epithelial and endothelial cells from the donor tissue before grafting, the grafted donor tissue actually contained only dead keratocyte cells in the stroma. The dead keratocytes in the graft may still have allogeneic immunogenicity, but they are too weak to induce immunologic inflammation and mild anti-inflammatory medications such as $0.1 \%$ fluorometholone eyedrops in short-term were sufficient to prevent this reaction. Based on the previous reports (Caldwell et al., 1984; Pettit, 1991) indicating that an allograft rejection rate of $10 \%-14 \%$ was found when fresh corneal tissue was used, we believe that cryopreserved donor tissue is more ideal than the fresh donor tissue.

Secondly, this technique can largely save donors.
The shortage of corneal donors is a critical problem. Since all cells will die in cryopreserved corneas, corneas with endothelial cells that are unsuitable for PK are still likely to be good as cryopreserved donor materials. Moreover, in ring-shaped peripheral DALK, all grafts were obtained from the residual cryopreserved corneoscleral rims after central keratoplasty. Therefore, this technique can make full use of donor corneas without requiring new materials.

Thirdly, the surgical procedure and postoperative treatment are much simpler. Since ring grafts were obtained from the residual corneoscleral rim, trephine was not needed in graft preparation (Cheng et al., 2005; Wang et al., 2012). We selected the matched corneoscleral rim according to the inside and outside diameters of the lesion before surgery and cut them into sizeable grafts during surgery. Since we prepared corneal beds almost to the Descemet's membrane, all grafts were taken as full-thickness without lamellar preparation procedures (Cheng et al., 2005). The only additional procedure was the removal of epithelial and endothelial cells by cellulose sponges. Therefore, the preparing of grafts was technically easier and less time-consuming. In addition, the postoperative treatment is also simple. As we mentioned before, $0.1 \%$ fluorometholone eyedrops were administered four times daily for six months to prevent mild postoperative inflammation, which can successfully avoid severe side-effects induced by long-term use of immunosuppressants (Wang et al., 2012). Except for transient ocular hypertension and drug-controlled secondary glaucoma which occurred occasionally, there were no other complications that needed to be managed. Therefore, our results showed this technique to be simple and successful.

Fourthly, in the inflammatory group, no inflammation recurrence was observed after the operation. For the inflammatory type TMD, cryopreserved donor material also avoided inflammation recurrence, which is of significant note, as a fresh donor may induce rejection and aggravate the inflammation. Therefore, cryopreserved donor material is safer than fresh donor material.

Wang et al. (2012) reported that no intraoperative corneal perforation occurs during LK surgery when guided by high-definition OCT and Pentacam imaging. In our study, maximal thinning was accurately judged by a slit-lamp and surgical microscope. 
Although we carefully tore off the epithelium at the thinnest point, perforation still occurred in $41.9 \%$ of the eyes. The thinnest point and lipid deposition were the most predilection sites of the perforation. These perforations originally existed just below the epithelium and thus their identification was inevitable during surgery. Incidence of these perforations was higher in the inflammatory group $(P=0.02)$. We speculated that lipid filtration may be caused by vascular congestion and increased vascular permeability, which may explain the greater lipid deposition and higher incidence of perforation in the inflammatory group. However, for these perforated eyes, there was no double anterior chamber. We speculated that this was due to the well-matched donor graft and recipient bed, and the relatively small size of the peripheral and ring-shaped graft. Since the exact reason was unknown, further research may be needed. There was also no complicated cataract and no delay in graft recovery. Unfortunately, graft clarity of the perforated eyes was worse than that of the non-perforated eyes.

In this study, high IOP was the most common complication, which was related to the range of the graft and intraoperative perforation. We hypothesize three causes for the high IOP: (1) the cryopreserved donor tissues were oedematous; (2) ring-shaped grafts were in the periphery and required two lines of sutures and these two factors crowded the peripheral anterior chamber; and (3) intraoperative perforation caused a shallow anterior chamber postoperatively. As a result, these three factors led to a high incidence (35.4\%) of high IOP. However, $90 \%$ of these cases $(10 / 11)$ were successfully controlled in $72 \mathrm{~h}$ with medication and secondary glaucoma was a rare occurrence (1/11).

The key goal of this surgery is to generate a more regular ocular surface and restore the integrity of the cornea at the same time. Theoretically, peripheral DALK can create normal corneal curvature and improve the visual acuity. Our results also showed that the postoperative BCVA of the ring-shaped group was good. However, both postoperative BCVA and astigmatism of the D-shaped group were poor. These results were consistent with those in previous reports (Cheng et al., 2005; Huang et al., 2008; Wang et al., 2012). We presume that in the D-shaped group, sutures near the optical zone led to a highly irregular astigmatism and decreased the visual acuity postop- eratively. Therefore, other appropriate surgical procedures should be considered over D-shaped DALK; enlarged grafts, such as whole corneal FBDLK, may be a more appropriate choice.

Taken together, this study manifested that peripheral DALK using a cryopreserved donor graft in treating TMD can reconstruct the corneal structure, regain normal corneal curvature, obtain better visual acuity, avoid allograft rejection, and make full use of donor corneas. However, D-shaped DALK did not achieve good visual outcomes and needs to be replaced by a more appropriate procedure.

\section{Compliance with ethics guidelines}

Dan HUANG, Wen-ya QIU, Bei ZHANG, Bing-hong WANG, and Yu-feng YAO declare that they have no conflict of interest.

All procedures followed were in accordance with the ethical standards of the responsible committee on human experimentation (institutional and national) and with the Helsinki Declaration of 1975, as revised in 2008 (5). Informed consent was obtained from all patients for being included in the study.

\section{References}

Alino, A.M., Perry, H.D., Kanellopoulos, A.J., et al., 1998. Conjunctival flaps. Ophthalmology, 105(6):1120-1123. [doi:10.1016/S0161-6420(98)96017-1]

Anderson, F.G.Jr., 1977. Repair of marginal furrow perforation. Ophthalmic Surg., 8:25-28.

Austin, P., Brown, S.I., 1981. Inflammatory Terrien's marginal corneal disease. Am. J. Ophthalmol., 92(2):189-192. [doi:10.1016/0002-9394(81)90768-6]

Brown, A.C., Rao, G.N., Aquavella, J.V., 1983. Peripheral corneal grafts in Terrien's marginal degeneration. Ophthalmic Surg., 14:931-934.

Caldwell, D.R., Insler, M.S., Boutros, G., et al., 1984. Primary surgical repair of severe peripheral marginal ectasia in Terrien's marginal degeneration. Am. J. Ophthalmol., 97(3):332-336. [doi:10.1016/0002-9394(84)90632-9]

Cheng, C.L., Theng, J.T., Tan, D.T., 2005. Compressive C-shaped lamellar keratoplasty: a surgical alternative for the management of severe astigmatism from peripheral corneal degeneration. Ophthalmology, 112(3):425-430. [doi:10.1016/j.ophtha.2004.10.033]

Doggart, J.H., 1930. Marginal degeneration of the cornea. $B r . J$. Ophthalmol., 14(10):510-516. [doi:10.1136/bjo.14.10.510]

Duke-Elder, S., 1965. System of Ophthalmology, Vol. 8, Part 2. Henry Kimpton, London, p.909-914.

Grayson, M., 1979. Disease of the Cornea. CV Mosby Co., St. Louis, p.189-191.

Guyer, D.R., Barraquer, J., McDonnell, P.J., et al., 1987. Terrien's marginal degeneration: clinicopathologic case reports. Graefe's Arch. Clin. Exp. Ophthalmol., 225(1): 19-27. [doi:10.1007/BF02155799]

Hanada, K., Iqarashi, S., Muramatsu, O., et al., 2008. Therapeutic keratoplasty for corneal perforation: clinical 
results and complications. Cornea, 27(2):156-160. [doi:10. 1097/ICO.0b013e31815b82f2]

Huang, T., Wang, Y., Ji, J., et al., 2008. Evaluation of different types of lamellar keratoplasty for treatment of peripheral corneal perforation. Graefe's Arch. Clin. Exp. Ophthalmol., 246(8):1123-1131. [doi:10.1007/s00417-008-0812-9]

Inq, J.J., Inq, H.H., Nelson, L.R., et al., 1998. Ten-year postoperative results of penetrating keratoplasty. Ophthalmology, 105(10):1855-1865. [doi:10.1016/S01616420(98)91030-2]

Iwamoto, T., DeVoe, A., Farris, R.L., 1972. Electron microscopy in cases of marginal degeneration of the cornea. Invest. Ophthalmol., 11(4):241-257.

Javadi, M.A., Karimian, F., Hosseinzadeh, A., et al., 2004. Lamellar crescentic resection for pellucid marginal corneal degeneration. J. Refract. Surg., 20(2):162-165.

Jonas, J.B., Rank, R.M., Budde, W.M., 2001. Tectonic sclerokeratoplasty and tectonic penetrating keratoplasty as treatment for perforated or predescemetal corneal ulcers. Am. J. Ophthalmol., 132(1):14-18. [doi:10.1016/S00029394(01)00874-1]

Kursiah, M.R., 2013. Iatrogenic corneal perforation in Terrien Marginal Degeneration. Med. J. Malaysia, 68(2):173-174.

Maccheron, L.J., Daya, S.M., 2012. Wedge resection and lamellar dissection for pellucid marginal degeneration. Cornea, 31(6):708-715. [doi:10.1097/ICO.0b013e31824 $000 \mathrm{e} 3]$

Panda, A., Sharma, N., Angra, S.K., et al., 1999. Therapeutic sclerokeratoplasty versus therapeutic penetrating keratoplasty in refractory corneal ulcers. Aust. N. Z. J. Ophthalmol., 27(1):15-19. [doi:10.1046/j.1440-1606.1999.00141.x]

Peleyer, U., Steuhl, K.P., Weidle, E.G., et al., 1992. Corneal graft rejection: incidence, manifestation, and interaction of clinical subtypes. Transplant Proc., 24(5):2034-2037.

Pettit, T.H., 1991. Corneoscleral freehand lamellar keratoplasty in Terrien's marginal degeneration of the cornealong-term results. Refract. Corneal Surg., 7(1):28-32.
Shimazaki, J., 2000. The evolution of lamellar keratoplasty. Curr. Opin. Ophthalmol., 11(4):217-223. [doi:10.1097/ 00055735-200008000-00002]

Soong, H.K., Farjo, A.A., Katz, D., et al., 2000. Lamellar corneal patch grafts in the management of corneal melting. Cornea, 19(2):126-134. [doi:10.1097/00003226-20000 3000-00002]

Varley, G.A., Macsai, M.S., Krachmer, J.H., 1990. The results of penetrating keratoplasty for pellucid marginal degeneration. Am. J. Ophthalmol., 110(2):149-152. [doi:10. 1016/S0002-9394(14)76983-1]

Wang, T., Shi, W., Ding, G., et al., 2012. Ring-shaped corneoscleral lamellar keratoplasty guided by highdefinition optical coherence tomography and Scheimpflug imaging for severe Terrien's marginal corneal degeneration. Graefe's Arch. Clin. Exp. Ophthalmol., 250(12):17951801. [doi:10.1007/s00417-012-2042-4]

Wu, S.Q., Zhou, P., Zhang, B., et al., 2012. Long-term comparison of full-bed deep lamellar keratoplasty with penetrating keratoplasty in treating cornealleucoma caused by herpes simplex keratitis. Am. J. Ophthalmol., 153(2):291-299.e2. [doi:10.1016/j.ajo.2011.07.020]

Yao, Y.F., 2008. A novel technique for performing full-bed deep lamellar keratoplasty. Cornea, 27(Suppl. 1):S19-S24. [doi:10.1097/ICO.0b013e31817f445f]

Yao, Y.F., Zhang, B., Zhou, P., et al., 2002. Autologous limbal grafting combined with deep lamellar keratoplasty in unilateral eye with severe chemical or thermal burn at late stage. Ophthalmology, 109(11):2011-2017. [doi:10.1016/ S0161-6420(02)01258-7]

Yao, Y.F., Jin, Y.Q., Zhang, B., et al., 2006. Recurrence of lattice corneal dystrophy caused by incomplete removal of stroma after deep lamellar keratoplasty. Cornea, 25(Suppl. 1):S41-S46. [doi:10.1097/01.ico.0000247212. 86014.35]

\section{中文概要:}

\section{本文题目：冰冻保存供体的周边深板层角膜移植治疗 Terrien's 角膜变性的临床疗效}

Peripheral deep anterior lamellar keratoplasty using a cryopreserved donor cornea for Terrien's marginal degeneration

研究目的: 观察采用冰冻保存供体的周边深板层角膜移植治疗 Terrien's 角膜变性（TMD）的临床疗效, 探讨 TMD 的最佳手术治疗方式。

创新要点: 供体角膜为冰冻保存, 其中部分为中央角膜移植术后剩余的角巩膜环, 并改进传统的手术方法。采用周 边深板层角膜移植，既充分利用和节约供体，避免排斥反应，又简化了手术操作过程，有效地恢复了眼 球完整性和提高术后视力。

研究方法: 研究对象为 1998 年 6 月至 2012 年 12 月期间在浙江大学医学院附属邵逸夫医院眼科中心进行周边深板层 角膜移植术的 27 例 TMD 病人的 31 只眼。根据病变范围和大小, 分别进行环形深板层角膜移植和 D 形深 板层角膜移植。所有植片均于 $-20{ }^{\circ} \mathrm{C}$ 冰冻保存, 冰冻保存的角巩膜环植片用于环形深板层角膜移植, 冰 冻保存的整个眼球用于 D 形深板层角膜移植。对所有术眼的病例资料、视频及影像学检查进行回顾性分 析，记录并总结患者的一般情况、术中表现、术后角膜结构的变化、屈光及视力的变化及术后并发症等。

重要结论: 采用冰冻保存供体的周边深板层角膜移植治疗 TMD 可以有效地恢复角膜结构的完整性且能长期维持 (图 3 和 4)。术后随访过程未发现免疫排斥反应, 环形深板层角膜移植术后视力较前明显提高, 而 3 例 ( $100 \%)$ $\mathrm{D}$ 形深板层角膜移植术后散光及视力均不提高 (表 1 和 2)。术前炎症及术中穿孔并不影响术后最终结果。

关键词组: Terrien's 角膜变性; 周边深板层角膜移植; 冰冻保存 